\title{
Video Segmentation Framework by Dynamic Background Modelling
}

\author{
Santiago Molina-Giraldo, Andres M. Álvarez-Meza, \\ Julio C. García-Álvarez, and Cesar G. Castellanos-Domínguez \\ Signal Processing and Recognition Group \\ Universidad Nacional de Colombia - sede Manizales \\ Campus La Nubia, km 7 via al Magdalena, Manizales-Colombia \\ \{smolinag, amalvarezme, jcgarciaa, cgcastellanosd\}@unal.edu.co
}

\begin{abstract}
Detecting moving objects in video streams is the first relevant step of information extraction in many computer vision applications, e.g. video surveillance systems. In this work, a video segmentation framework by dynamic background modelling is presented. Our approach aims to update suitably the background model of a scene that is recorded by a static camera. For such purpose, we develop an optical flow based methodology to suitable track moving objects, which can stop or change smoothly their movement along the video. Moreover, a light variations identification stage, is employed to avoid possible confusions between illumination changes and objects in movement. Regarding this, our approach is able to ensure a suitable background modelling in real world scenarios. Attained results show that our framework outperforms, in well-known datasets, state of the art methodologies.
\end{abstract}

Keywords: background subtraction, optical flow, tracking.

\section{Introduction}

A system that monitors an area by camera and is able to detect and track moving objects is called a surveillance system. Intelligent video surveillance systems can achieve unsupervised results using video segmentation, where the moving objects are extracted from video sequences. Nevertheless, the challenge is to devise and implement automatic systems able to perform both activities, detection and tracking. Afterwards, the main goal is to interpret their activities and behaviours to support computer vision analysis (e.g. object classification, tracking, activity understanding, among others) [8, 9]. In this regard, it is necessary to segment (subtract) each object from the scene to facilitate such kind of analysis.

Our main goal is on the detection phase of a general visual surveillance system using static cameras. Aside from the intrinsic usefulness of being able to segment video streams into foreground and background components, detecting moving objects provides a focus of attention for recognition, classification, and activity analysis, making these later steps more efficient [3]. Many segmentation algorithms have been proposed, among them, algorithms with background modelling usually show superior performance, by exploiting the prior information about the process, specially, for static 
cameras environments [2]. Thus, background subtraction, that consists in maintaining an updated model of the background and detecting (segmenting) moving objects as those that deviate from such a model. Given a suitable background model, the system is able to subtract it, as a typical and crucial process for a surveillance system to detect moving objects that may enter, leave, move or left unattended in the surveillance region.

Compared to other approaches, such as optical flow, background modelling is computationally affordable for real-time applications [1]. The main problem is its sensitivity to dynamic scene changes, and the consequent need for the background model adaptation via background maintenance. Such problem is known to be significant and difficult [3]. Furthermore, image sequences with dynamic backgrounds often cause false classification of pixels (e.g. light variations). One common solution is to map the input samples into alternate color spaces, however, it has failed to solve this problem and an enhanced solution is the use of image features, where the distributions at each pixel may be modelled in a parametric manner using a mixture of Gaussians or by using nonparametric kernel density estimation [5,7]. The self organizing maps have been also explored as an alternative for the background subtraction task, because of their nature to learn by means of local variations [8]. However, these techniques have some drawbacks when dealing with the actualization of the background model, when in the video sequence there are objects which after moving become static.

In this work, a video segmentation framework by dynamic background modelling is presented. Our approach aims to update the model of a scene that is recorded by a static camera. In this regard, an optical flow based methodology is proposed to suitable track moving objects, which can stop or change smoothly their movement along the video. Objects detected as static are stored into a memory, with the purpose of not updating the model in these regions. Moreover, a light variations identification stage, is proposed to avoid possible confusions between illumination changes and objects in movement. Thus, our framework is able to ensure a suitable background modelling in real-world scenarios. The remainder of this work is organized as follows. In section 2 , the proposed framework is described. In section 3, the experiments and results are presented. Finally, in sections 4 and 5 we discuss and conclude about the attained results.

\section{Theoretical Background}

\subsection{Region Change Detection}

Let $\boldsymbol{\Phi}=\left\{\boldsymbol{F}^{(t-T)}, \boldsymbol{F}^{(t-T+1)}, \ldots, \boldsymbol{F}^{(t)}\right\}$ be a set of images of a given video, with $t=T+1, \ldots, \infty$ and $\boldsymbol{F}^{(t)} \in \mathbb{R}^{h \times w \times N_{c}}$. Here, $h$ is the number of rows of the frame, $w$ the number of columns, and $N_{c}$ the number of color channels. Given the frame $\boldsymbol{F}^{(t)}$, the mean intensity matrix $\boldsymbol{M}^{(t)} \in \mathbb{R}^{h \times w}$, with elements $M_{i, j}^{(t)}=\boldsymbol{E}\left\{\boldsymbol{f}_{(i, j)}^{(t)}\right\}$, where $\boldsymbol{f}_{(i, j)}^{(t)} \in \mathbb{R}^{1 \times N_{c}}$ is a row vector containing the $N_{c}$ color channels of pixel $(i, j)$ and notation $\boldsymbol{E}\{\cdot\}$ stands for expectation operator. The purpose is to detect changes between the frame $t$ and $t-1$, then, $\boldsymbol{M}^{(t)}$ is divided into $n_{p}=h \times w$ patches, and the Sum of Absolute Differences (SAD) matrix $\boldsymbol{H}^{(t)} \in \mathbb{R}^{h \times w}$ is calculated as 


$$
H_{i, j}^{(t)}= \begin{cases}1 & \left\|\Omega_{(i, j)}^{(t)}-\Omega_{(i, j)}^{(t-1)}\right\|_{F}^{2}<\xi_{H} \\ 0 & \text { Otherwise }\end{cases}
$$

where the patch $\Omega_{(i, j)}^{(t)} \in \mathbb{R}^{h_{p} \times w_{p}}$ contains the spatial neighbourhood of the pixel $(i, j)$ in $M^{(t)}, \xi_{H} \in \mathbb{R}^{+}$is a given threshold parameter, and $\|\cdot\|$ is the Frobenius norm operator. Thus, matrix $\boldsymbol{H}^{(t)}$ in (1) highlights pixels detected as moving from frame $t-1$ to $t$.

\subsection{Motion Modeling by Optical Flow}

Now the challenge is to detect the apparent movement of the regions detected as moving in matrix $\boldsymbol{H}^{(t)}$, in this regard, an optical flow procedure is employed. By definition, an optical flow field is a vector field that describes the velocity of pixels in an image sequence. Due to the complexity of most of the optical flow approaches proposed in the state of the art [4, 10], we propose to use a selective optical flow approach based on block matching, where only for the patches highlighted as moving in $\boldsymbol{H}^{(t)}$ the optical flow is computed. Given the patch $\Omega_{(i, j)}^{(t-1)}$ in $\boldsymbol{M}^{(t-1)}$ marked as moving, $\boldsymbol{M}^{(t)}$ is used to compute the optical flow by searching in a neighbourhood of size $\kappa$ around $(i, j)$ the patch that best matches $\Omega_{(i, j)}^{(t-1)}$ by using the SAD. Then, the relative coordinates $(i+v i, j+v j)$ of such patch are stored in matrices $\boldsymbol{X}^{(t)} \in \mathbb{R}^{h \times w}$ and $\boldsymbol{Y}^{(t)} \in \mathbb{R}^{h \times w}$ respectively. Hence, the apparent movement from frame $t-1$ to $t$, is described by matrices $\boldsymbol{X}^{(t)}$ for $x$ coordinates and $\boldsymbol{Y}^{(t)}$ for $y$ coordinates.

\subsection{Object Movement Identification and Static Object Memory Computation}

Given the motion matrix $\boldsymbol{H}^{(t-1)}$, some morphological filters are applied to achieve a smooth representation of the movement. Furthermore, above procedure allows to enhance each object shape representation. Afterwards, the filtered version of $\boldsymbol{H}^{(t-1)}$ is processed to highlight connected components, thus is, a set of objects $\boldsymbol{\Xi}^{(t-1)}=$ $\left\{\boldsymbol{\Theta}_{1}^{(t-1)}, \boldsymbol{\Theta}_{2}^{(t-1)}, \ldots, \boldsymbol{\Theta}_{l_{t-1}}^{(t-1)}\right\}$ is inferred by an 8 -connected based operation, where $\boldsymbol{\Theta}_{l_{t-1}}^{(t-1)}$ is a real-valued matrix of size $h_{l_{t-1}}^{(t-1)} \times w_{l_{t-1}}^{(t-1)}$. For each detected object, the mode direction vector $\boldsymbol{\mu}_{l_{t-1}}^{(t-1)} \in \mathbb{R}^{2 \times 1}$ is estimated, where the first and second components of $\boldsymbol{\mu}_{l_{t-1}}^{(t-1)}$ correspond to the mode of pixels $(i, j)$ in $\boldsymbol{X}^{(t-1)}$ and $\boldsymbol{Y}^{(t-1)}$ that are contained in object $\boldsymbol{\Theta}_{l_{t-1}}^{(t-1)}$. In addition, the magnitude of $\boldsymbol{\mu}_{l_{t-1}}^{(t-1)}$ is computed and stored into $v_{l_{t-1}}^{(t-1)} \in \mathbb{R}^{+}$. So, we are looking for the trend of motion direction for each detected object. Then, each object $\Theta_{l_{t-1}}^{(t-1)} \subset \boldsymbol{H}^{(t-1)}$ is enclosed by a bounding box. Such box is moved according to $\boldsymbol{\mu}_{l_{t-1}}^{(t-1)}$ and $v_{l_{t-1}}^{(t-1)}$, and it is mapped into $\boldsymbol{H}^{(t)}$. After that, the number of projected pixels $\lambda_{l_{t-1}}^{(t)} \in \mathbb{N}$ at instant $t$, which are contained into the mapped box are calculated. Object $\Theta_{l_{t-1}}^{(t-1)}$ is detected as moving from $t-1$ to $t$ if 


$$
\frac{\lambda_{l_{t}}^{(t)}}{\lambda_{l_{t-1}}^{(t)}}>\xi_{\lambda}
$$

with $\xi_{\lambda} \in \mathbb{N}$. Otherwise, if (2) is not accomplished, object $\boldsymbol{\Theta}_{l_{t-1}}^{(t-1)}$ is labelled as static. Hence, the static objects set $\boldsymbol{\Psi}^{(t)}$ is composed by the $\boldsymbol{\Theta}_{l_{t-1}}^{(t-1)}$ objects that are labelled as static at instant $t$. So, a static memory matrix $\boldsymbol{B}^{(t)} \in \mathbb{R}^{h \times w}$ can be written as

$$
B_{i, j}^{(t)}= \begin{cases}1 & (i, j) \in \bigcup_{t}^{t_{i}=1} \Psi^{\left(t_{i}\right)} \\ 0 & \text { Otherwise }\end{cases}
$$

Note that, a static object in $\boldsymbol{\Psi}^{(t)}$ only can be considered as moving, if at a posterior time instant the condition (2) is accomplished, then, removing it from the set.

\subsection{Background Modeling Updating}

The main goal of our approach is to deal with real-world environment conditions, looking for a background model that allows to identify, as well as possible, smooth movements, which are inherent to the scene. Hence, we propose to define a Gaussianbased background model $\zeta\left(\boldsymbol{\Gamma}^{(t-1)}, \boldsymbol{\Sigma}^{(t-1)}\right)$, with $\boldsymbol{\Gamma}^{(t-1)} \in \mathbb{R}^{h \times w \times N_{c}}$ and $\boldsymbol{\Sigma}^{(t-1)} \in$ $\mathbb{R}^{h \times w \times N_{c}}$, as in (4)

$$
p(i, j, z)=\exp \left(-\frac{\left\|f_{i, j, z}^{(t-1)}-\Gamma_{i, j, z}^{(t-1)}\right\|_{2}^{2}}{2 \Sigma_{i, j, z}^{(t-1)^{2}}}\right),
$$

being $\Gamma_{i, j, z}^{(t-1)} \in \mathbb{R}^{+}$the background model value of pixel $(i, j)$ at time instant $t-1$ in channel $z$, and $\Sigma_{i, j, z}^{(t-1)} \in \mathbb{R}^{+}$a color band-width value.

Thus, in order to update the parameters of the model, the mean color value matrix $\boldsymbol{M}^{(t)} \in \mathbb{R}^{h \times w \times N_{c}}$ is defined as

$$
M_{i, j, z}^{(t)}=\boldsymbol{E}\left\{\boldsymbol{v}_{i, j, z}^{(t)}\right\}
$$

being $\boldsymbol{v}_{i, j, z}^{(t)}=\left\{\boldsymbol{F}_{i, j, z}^{(t)}, \boldsymbol{F}_{i, j, z}^{(t-1)}, \ldots, \boldsymbol{F}_{i, j, z}^{(t-T)}\right\}$. Similarly, a deviation matrix $\boldsymbol{D}^{(t)} \in$ $\mathbb{R}^{h \times w \times N_{c}}$ is computed as

$$
D_{i, j, z}^{(t)}=\sigma\left\{\boldsymbol{v}_{i, j, z}^{(t)}\right\}
$$

where $\sigma\{\cdot\}$ is the deviation operator. Finally, the Gaussian-based background model $\zeta\left(\Gamma^{(t)}, \boldsymbol{\Sigma}^{(t)}\right)$ is updated as in (7) and (8)

$$
\Gamma_{i, j, z}^{(t)}= \begin{cases}M_{i, j, z}^{(t)} & \left(D_{i, j, z}^{(t)}<\Sigma_{i, j, z}^{(t-1)}\right) \cap\left(B_{i, j}^{(t)}=0\right) \\ \Gamma_{i, j, z}^{(t-1)} & \text { Otherwise }\end{cases}
$$




$$
\Sigma_{i, j, z}^{(t)}= \begin{cases}D_{i, j, z}^{(t)} & \left(D_{i, j, z}^{(t)}<\Sigma_{i, j, z}^{(t-1)}\right) \cap\left(B_{i, j}^{(t)}=0\right) \\ \Sigma_{i, j, z}^{(t-1)} & \text { Otherwise }\end{cases}
$$

It is important to note that the matrices $\boldsymbol{M}^{(t)}$ and $\boldsymbol{D}^{(t)}$ are computed only by using the last $T$ frames, then it is only needed to store these frames for such computation. Given the provided background model $\zeta\left(\boldsymbol{\Gamma}^{(t)}, \boldsymbol{\Sigma}^{(t)}\right)$, a clustering based segmentation algorithm as described in [6] is used to extract the moving objects from the scene. This approach propose to use kernel representations and a relevance analysis in order to conform an enhanced feature space, then a tuned kmeans algorithm with 2 groups is performed over the enhanced space, aiming to group static pixels in one cluster and moving pixels in the other. Thence, a segmented binary matrix $\boldsymbol{S}^{t}$ is attained.

\subsection{Illumination Change Detection}

Nonetheless, some scene conditions (e.g. light variations, casted shadows) perturb the quality of the segmented image. Therefore, it is proposed to debug the matrix $\boldsymbol{S}^{(t)}$ by means of an illumination change detection stage. For such purpose, we propose to use the normalized RGB representation as studied in [5]. Then a normalized channel difference matrix $\boldsymbol{A}_{\boldsymbol{n}}{ }^{(t)} \in \mathbb{R}^{h \times w}$ is calculated as

$$
A_{n i, j}^{(t)}= \begin{cases}1 & \prod_{z=1}^{N_{c}} \eta\left(f_{n_{i, j, z}}^{(t)}-\Gamma_{n_{i, j, z}}^{(t-1)}, \xi_{A_{n}}\right)=1 \\ 0 & \text { Otherwise }\end{cases}
$$

where

$$
f_{n i, j, z}^{(t)}=\frac{f_{i, j, z}^{(t)}}{\sum_{r=1}^{N_{c}} f_{i, j, r}^{(t)}} \quad \Gamma_{n_{i, j, z}}^{(t-1)}=\frac{\Gamma_{i, j, z}^{(t-1)}}{\sum_{r=1}^{N_{c}} \Gamma_{i, j, r}^{(t-1)}}
$$

are the normalized color channel $z$ of pixel $(i, j)$ in $\boldsymbol{F}^{(t)}$ and $\boldsymbol{\Gamma}^{(t-1)}$, respectively. And $\eta\left(f_{n_{i, j, z}}^{(t)}-\Gamma_{n_{i, j, z}}^{(t-1)}, \xi_{A}\right)=1$, if $\left\|f_{n_{i, j, z}}^{(t)}-\Gamma_{n_{i, j, z}}^{(t-1)}\right\|_{2}^{2}<\xi_{A}$. Similarly, a difference matrix $\boldsymbol{A}_{\boldsymbol{n}}{ }^{(t)} \in \mathbb{R}^{h \times w}$ is calculated as

$$
A_{i, j}^{(t)}= \begin{cases}1 & \prod_{z=1}^{N_{c}} \eta\left(f_{i, j, z}^{(t)}-\Gamma_{i, j, z}^{(t-1)}, \xi_{A}\right)=1 \\ 0 & \text { Otherwise }\end{cases}
$$

Thus, $\boldsymbol{A}_{\boldsymbol{n}}{ }^{(t)}$ in (9) looks for chromaticity changes in pixels by using a normalized color space computed according to (10), this help to suppress the shadows casted by moving objects. Nonetheless, the lightness representation is lost by using only this information, in this regard, $\boldsymbol{A}^{(t)}$ in (11) is employed. Then, combining $\boldsymbol{A}^{(t)}, \boldsymbol{A}_{\boldsymbol{n}}{ }^{(t)}$, and $\boldsymbol{S}^{(t)}$, it is possible to attain a correct segmented matrix $\hat{\boldsymbol{S}}^{(t)} \in \mathbb{R}^{h \times w}$ avoiding illumination changes as

$$
\hat{S}_{i, j}^{(t)}=S_{i, j}^{(t)} A_{i, j}^{(t)} A_{n_{i, j}}^{(t)}
$$




\section{Experiments}

The proposed methodology for automatic video segmentation is tested using some well known databases of the state of the art. Moreover, a traditional video segmentation algorithm named Self-Organizing Approach to Background Subtraction (SOBS), which builds a background model by learning in a self-organizing manner the scene variations, is employed as benchmark [8]. Each database includes image sequences that represent typical situations for testing video surveillance systems.

\subsection{Databases}

A-Star-Perception 1]: This database contains 9 image sequences recorded in different scenes. Hand-segmented ground truths are available for each sequence, thus, supervised measures can be used. For concrete testing, the sequences: Water-Surface, Fountain, Shopping-Mall, and Hall are used. The first two sequences are recorded in outdoor scenarios which present high variations due to their nature. Hence, the segmentation process poses a considerable challenge. The other two sequences are recorded in public halls, in which are present many moving objects casting strong shadows crossing each other, being difficult to segment.

Left-Packages2: It contains 5 different image sequences recorded at an interior scenario, which has several illumination changes. The main purpose of this database is the identification of abandoned objects (a box and a bag). For testing, hand-segmented ground truths from randomly selected frames are made.

MSA 3 : It contains a single indoor sequence with stable lighting conditions. Nonetheless, strong shadows are casted by the moving objects. The purpose of this sequence is the detection of an abandoned briefcase. Hand-segmented ground truths from randomly selected frames are made in order to give a quantitative measure.

\subsection{Experimental Set-Up and Results}

For all the experiments, the free parameters above mentioned (section 2) are set as: $T=15, \xi_{H}=\left(h_{p} \times w_{p} * 15\right)^{2}, \xi_{A}=75, \xi_{A_{n}}=0.05, \xi_{\lambda}=0.5$. The SOBS algorithm is free available 4 , and for testing the parameters were left as default. For measuring the accuracy of the methodologies, three different pixel-based measures have been adopted, as described in (13), (14), and (15)

$$
\begin{array}{r}
\text { Recall }=t_{p} /\left(t_{p}+f_{n}\right) \\
\text { Precision }=t_{p} /\left(t_{p}+f_{p}\right) \\
\text { Similarity }=t_{p} /\left(t_{p}+f_{n}+f_{p}\right),
\end{array}
$$

\footnotetext{
${ }^{1}$ http://perception.i2r.a-star.edu.sg

2 http: / / homepages.inf.ed.ac.uk/rbf/CAVIARDATA1

3 http: / / cvprlab.uniparthenope

4 http://www.na.icar.cnr.it/ maddalena.1/

MODLab/SoftwareSC-SOBS.html
} 
where $t_{p}$ (true positives), $f_{p}$ (false positives) and $f_{n}$ (false negatives) are obtained while comparing against a hand-segmented ground truth [8]. A method is considered good if it reaches high Recall measures, without sacrificing Precision. Similarity is adopted aiming to further compare the results achieved by other proposed algorithms.

In Table 1 are presented the computed measures for the proposed approach and the SOBS algorithm [8]. Additionally, in Fig. 1 an example of the proposed motion direction calculation stage is presented. Fig. 2] shows an example of the proposed stage to detect static objects. Finally, Fig. 3 and Fig. 4 present some relevant segmentation results related to the proposed algorithm and the SOBS method.

Table 1. Segmentation performance for the proposed approach and SOBS

\begin{tabular}{l|l|l|l|l|l|l}
\hline & \multicolumn{3}{|c|}{ Proposed approach } & \multicolumn{3}{c}{ SOBS } \\
\hline Video & Recall & Precision & Similarity & Recall & Precision & Similarity \\
\hline Water-Surface & $0.968 \pm 0.027$ & $0.863 \pm 0.067$ & $0.837 \pm 0.054$ & $0.709 \pm 0.103$ & $0.998 \pm 0.143$ & $0.708 \pm 0.129$ \\
Shopping-Mall & $0.771 \pm 0.144$ & $0.632 \pm 0.070$ & $0.543 \pm 0.123$ & $0.522 \pm 0.192$ & $0.861 \pm 0.126$ & $0.482 \pm 0.143$ \\
MSA & $0.989 \pm 0.005$ & $0.762 \pm 0.026$ & $0.756 \pm 0.028$ & $0.778 \pm 0.053$ & $0.788 \pm 0.042$ & $0.748 \pm 0.045$ \\
Left-Bag & $0.735 \pm 0.147$ & $0.863 \pm 0.056$ & $0.657 \pm 0.126$ & $0.472 \pm 0.111$ & $0.642 \pm 0.122$ & $0.373 \pm 0.116$ \\
Left-Box & $0.914 \pm 0.066$ & $0.891 \pm 0.039$ & $0.823 \pm 0.075$ & $0.746 \pm 0.108$ & $0.806 \pm 0.093$ & $0.634 \pm 0.104$ \\
\hline
\end{tabular}

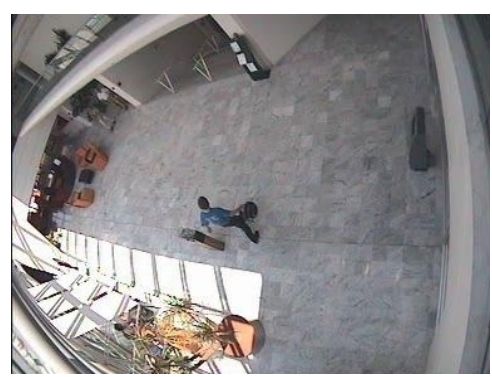

(a) A Left-Bag video frame

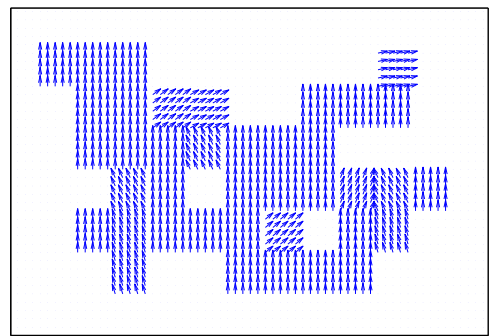

(c) Optical flow calculation (zoom over moving pixels)

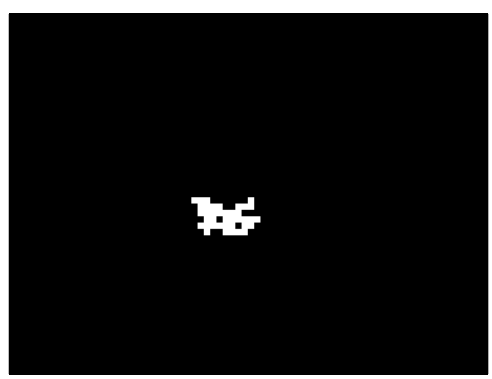

(b) Detected moving pixels (white)

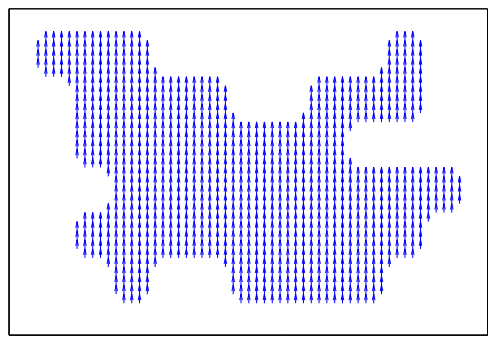

(d) Optical flow mode (zoom over moving pixels)

Fig. 1. Motion detection results over Left-Bag video 


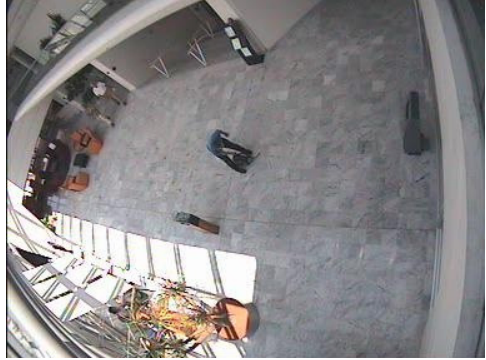

(a) Left-Bag video (frame 45)

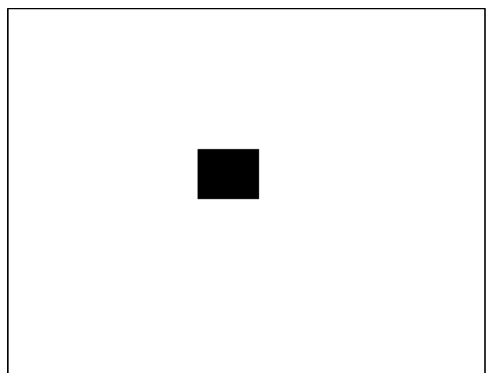

(c) Estimated static region

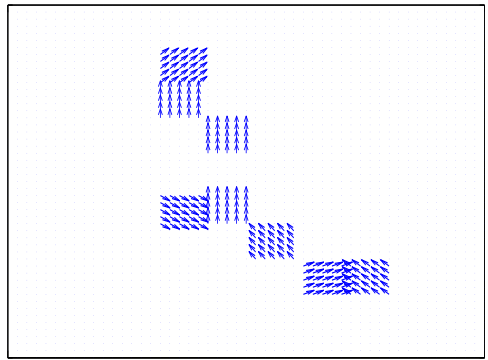

(b) Optical flow calculation

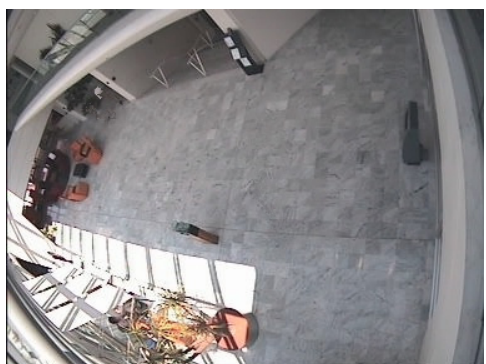

(d) Background model

Fig. 2. Detection of static object after movement (Left-bag video example)

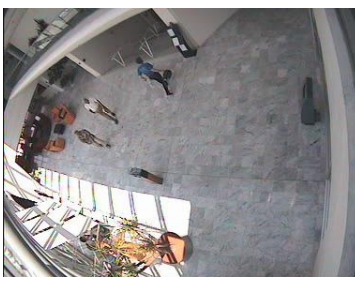

(a) Frame 155

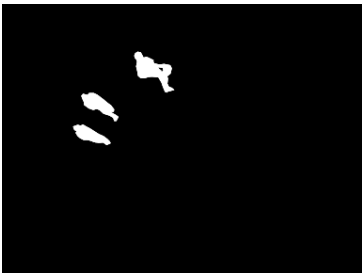

(d) Ground truth

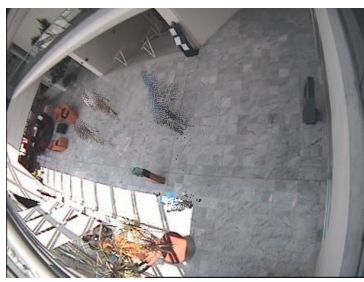

(b) SOBS background model

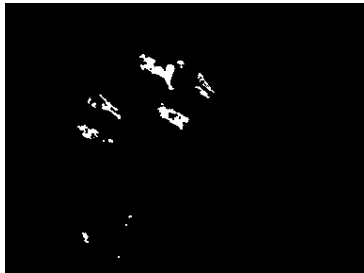

(e) SOBS segmentation

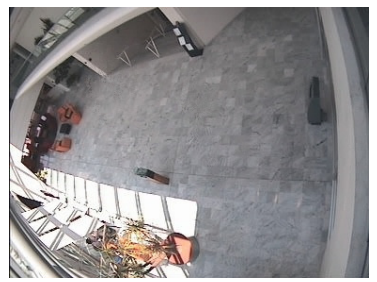

(c) Proposed approach background model

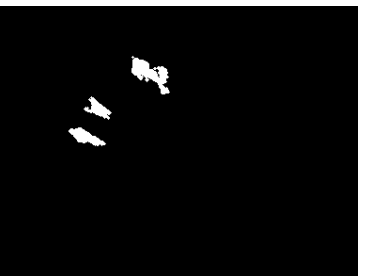

(f) Proposed approach segmentation

Fig. 3. Segmentation results for SOBS and the proposed approach 


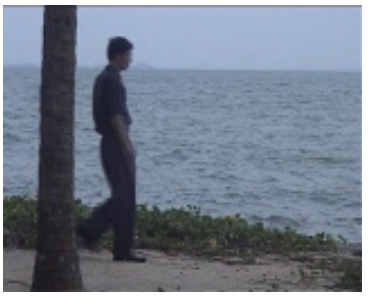

(a) Water-Surface frame (b) 1509

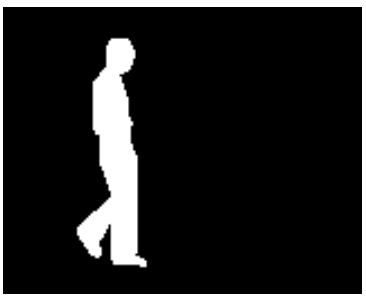

(d) Ground truth

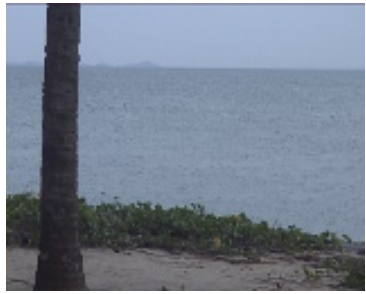

background model

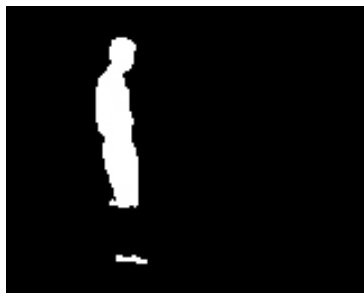

(e) SOBS segmentation

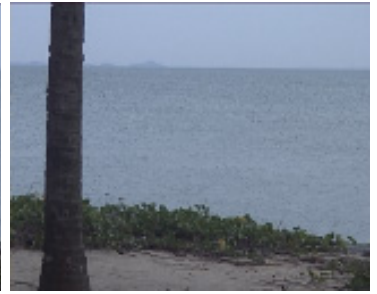

(c) Proposed approach background model

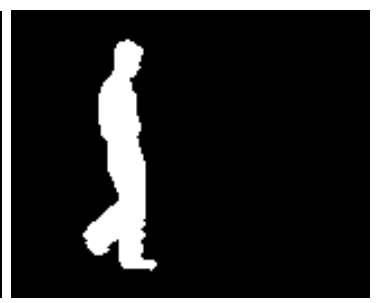

(f) Proposed approach segmentation

Fig. 4. Segmentation results for SOBS and the proposed approach

\section{Discussion}

From Fig 1 it can be noticed the attained results for background modeling of frame 17 in video Left-Bag. Moreover, in Fig. 1(b) is showed that our framework is able to identify pixels that are moved from frame 16 to 17 . Given the optical flow calculation (movement direction), a zoom over the pixels that are detecting as movement, and the optical flow direction as computed as shown in Fig. 1 (c), where it can be perceived a motion direction trend. Above mentioned trend is suitable softened by our approach (see1(d)). Thus, our framework is able to track the motion direction of a given object.

Now, from Fig. 2, it is presented the background modelling of video Left-Bag, where a person who had been previously walking stops at frame 45. In this regard, Fig. 2(b) describes the detected motion pixels, however, these pixels do not exhibit a clear direction of movement. Consequently, our approach identifies such region as an static object (see Fig. 2(c)). So, the background model at frame 45 is suitable inferred, as can be visually corroborated in Fig. 22(d).

In addition, in Figs. 3 and 4 some relevant segmentation results are showed. As can be seen from Figs. 3 (f) and 4 (f) proposed framework obtains suitable segmentation results, while the SOBS approach is not able to deal with light changes, moving/stopping objects, difficult moving trajectories, and/or camouflages, as seen in 3(d) and 4(d). Furthermore, Figs. 3 (e) and 4(e) prove how our framework suitable updates the background modelling, even against real-world video conditions (as mentioned above). Otherwise, SOBS approach do not obtain an appropriated background modelling for all the provided cases (see Figs. 3(c) and 4(c)). The above statements are corroborated by the 
attained segmentation performance shown in Table 1 Overall, the proposed approach outperforms the state of the art benchmark over the analysed video sequences.

\section{Conclusions}

A video segmentation framework by dynamic background modelling was presented. We developed an optical flow based methodology to take into account object trajectories into the background model. Thus, it is possible to identify when an object in movement stops or changes smoothly their motion along the video, giving a memory property to our model, then the model is updated only when needed. Moreover, a light variations identification stage is employed, which allows to avoid confusions between illumination changes and objects in movement. Hence, our approach aims to update suitable the background model of an scene that is recorded by a static camera. Attained results showed that our framework outperforms, in well-known datasets, state of the art methodologies, being suitable for supporting real video surveillance applications. As future work, it would be interesting to consider other kind of optical flow based models to deal with occluded objects and the inclusion of a multimodal background model. Furthermore, the proposed approach would be implemented as a real-time application.

\section{References}

1. Barron, J.L., Fleet, D.J., Beauchemin, S.: Performance of optical flow techniques. International Journal of Computer Vision 12(1), 43-77 (1994)

2. Chen, T.W., Hsu, S.C., Chien, S.Y.: Robust video object segmentation based on k-means background clustering and watershed in ill-conditioned surveillance systems. In: 2007 IEEE International Conference on Multimedia and Expo, pp. 787-790 (July 2007)

3. Collins, R.T., Lipton, A., Kanade, T., Fujiyoshi, H., Duggins, D., Tsin, Y., Tolliver, D., Enomoto, N., Hasegawa, O., Burt, P., et al.: A system for video surveillance and monitoring, vol. 102. Carnegie Mellon University, the Robotics Institute Pittsburg (2000)

4. Correia, M.V., Campilho, A.C.: Real-time implementation of an optical flow algorithm. In: Proceedings of the Pattern Recognition, vol. 4, pp. 247-250. IEEE (2002)

5. Elgammal, A., Duraiswami, R., Harwood, D., Davis, L.: Background and foreground modeling using nonparametric kernel density estimation for visual surveillance. Proceedings of the IEEE 90(7), 1151-1163 (2002)

6. Molina-Giraldo, S., Carvajal-Gonzales, J., Álvarez-Meza, A., Castellanos-Domínguez, G.: Video segmentation based on multi-kernel learning and feature relevance analysis for object classification. In: ICPRAM (2013)

7. Klare, B., Sarkar, S.: Background subtraction in varying illuminations using an ensemble based on an enlarged feature set. In: IEEE Computer Society Conference on CVPR Workshops 2009, pp. 66-73 (June 2009)

8. Maddalena, L., Petrosino, A.: A self-organizing approach to background subtraction for visual surveillance applications. IEEE Transactions on Image Processing 17(7), 1168-1177 (2008)

9. Raty, T.: Survey on contemporary remote surveillance systems for public safety. IEEE Transactions on Systems, Man, and Cybernetics 40(5), 493-515 (2010)

10. Zhou, D., Zhang, H.: Modified gmm background modeling and optical flow for detection of moving objects. In: 2005 IEEE International Conference on Systems, Man and Cybernetics, vol. 3, pp. 2224-2229. IEEE (2005) 